\title{
Advancement of technology and human being: a little comparison between human and human robot
}

\begin{abstract}
In the article "advancement of technology and human being: a little comparison between human and human robot" the author elaborate the history of human robots and then explained that how human robot is differ from human. They also explained that which one is better from both why we need for human being ever. This is basically a review report article because authors' observations and study depend on existing articles and reports. They driven the data from internet and prepared a review article. This article gives concise detail on Robots and their development and then it provide the advantages and disadvantages of robots. At the end author conclude that there is always the need of human being because robots' intelligence is not working as human brain
\end{abstract}

Keywords: robot, humanoid, human being, advance life, era of advancement, technological achievements, comparison report, need of human being
Volume 4 Issue 5 - 2018

\author{
Adila Kokab,' Fakhra Umbreen, ${ }^{2}$ Misbah \\ Roohi $^{3}$ \\ 'Teacher at Education Department, Pakistan \\ 2Lecturer, Education department, Ibn-e-Ameer College, Pakistan \\ IIndependent Researcher, Pakistan
}

\begin{abstract}
Correspondence: Adila Kokab, Teacher at Education Department, Village Kasab P.O Box Chak Kamala Tehsil \& District Gujrat,Village: Kasab, Pakistan, Tel 00923364982217 , Email adilakikab@gmail.com
\end{abstract}

Received: January 01, 2018 | Published: October 09, 2018

\section{Introduction}

This is the era of technology advancement especially in recent decade's robot technology grown faster than last decades. In these some decades human introduce the Human robot which seems like to be human. These types of robots can do tasks of daily life and can work easily in some area of industry, just like human being. The robots that are seemed to be human have their own history and way of development. Human being always trying to shaped robots in their own image. Since the very first Robot description of an automaton in a Daoist form the fifth century B.C to the latest Robot in 2017. A humanoid robot is a robot with its body shape worked to look like the human body. The plan might be for useful purposes, for example, collaborating with human instruments and conditions, for trial purposes, for example, the investigation of al movement, or for different purposes. When all is said in done, humanoid robots have middle, a head, two arms, and two legs, however a few types of humanoid robots may display just piece of the body, for instance, from the midriff up. Some humanoid robots additionally have makes a beeline for reproduce human facial highlights, for example, eyes and mouths. Androids are humanoid robots worked to tastefully take after people. Humanoid robots are currently utilized as an examination instrument in a few logical zones. Scientists need to comprehend the human body structure and conduct (biomechanics) to assemble and think about humanoid robots. On the opposite side, the endeavor to reenact the human body prompts a superior comprehension of it. Human insight is a field of study which is centered on how people gain from tangible data keeping in mind the end goal to get perceptual and engine aptitudes. This learning is utilized to create computational models of human conduct and it has been enhancing after some time. It has been proposed that extremely propelled apply autonomy will encourage the upgrade of conventional people. In spite of the fact that the underlying point of humanoid investigate was to manufacture better orthosis and prosthesis for people, information has been exchanged between the two controls. A couple of cases are fueled leg prosthesis for neuro muscularly hindered, lower leg foot orthosis, organic practical leg prosthesis and lower arm prosthesis. Other than the examination, humanoid robots are being created to perform human assignments like individual help, where they ought to have the capacity to help the wiped out and elderly, and messy or perilous occupations. Humanoids are likewise appropriate for some standard employments, for example, assistant and laborer in a car producing line. Fundamentally, since they can utilize apparatuses and work hardware and vehicles intended for the human frame, humanoids could hypothetically play out any assignment an individual can, inasmuch as they have the best possible programming. Notwithstanding, the many-sided quality of doing as such is tremendous. ${ }^{1}$

They are additionally winding up progressively well known as performers. For instance, Ursula, a female robot, sings, plays music, moves, and addresses her groups of onlookers at all inclusive Studios. A few Disney amusement stop indicates utilization of animatrons, robots that look, move, and talk much like individuals. They look so sensible that it can be difficult to disentangle from a separation regardless of whether they are really human. In spite of the fact that they have a reasonable look, they have no comprehension or physical self-rule. Different humanoid robots and their conceivable applications in day by day life are highlighted in a free narrative film called Fitting and Implore, which was discharged in 2010. Humanoid robots, particularly those with counterfeit consciousness calculations, could be helpful for future hazardous or potentially inaccessible space investigation missions, without wanting to turn back around again and come back to Earth once the mission is completed. So the robots can do all tasks that human being performed. ${ }^{2}$

\section{Objectives of the study}

In the current study authors have some purpose those are:

a. To know the brief history of human robots

b. To investigate the difference between human robots and human being

c. To elaborate the disadvantage of human robots if any

\section{Significance of the study}

There are a lot of studies on Robots, robots advancements and effectiveness of robots in daily life or in industrial life. But there is no study which describes the difference between human and human 
robots. That's why authors select this topic because it is very important to describe the difference between both. Because mostly the new generation look to be very impressed by Robots and thought that there is no need of human if they have robots in their life. So the current study is very important because it elaborate the whole phenomenon about robot and human's difference and why there is still need for human being in the world?

\section{Discussion}

The Human Robots are designed just like human being. They are flexible so they can move easily for doing any task and for their own safety. The latest robots have some feelings in their face expressions just like smile etc. These robots perform many tasks like driving, cooking, talking, walking, providing entertainment and also can fulfill all daily cores that any human can did or perform. But the other side these robots not actually the human beings because after all these are only just like the machines which is programmed and designed by human. These robots that having human body also has their own advantages and disadvantages.

\section{Advantages of robots}

Robots have many advantages for human life. Those are described as:

a. Robots can work for 24 hours and seven days without getting tired and bored.

b. Robots are more brave than human so they can visit that places where human feel scared while visiting or work there. For example in the space, on that plants where human cannot survived or in horrible places etc.

c. Robots can work without salary and food and also accomplished very difficult tasks that human not performed well.

d. Robots accomplished their tasks more faster and accurately than human beings

e. Robots can entertain the people without their own interest and played very helpful role for the depression's patient.

f. Robots can work without feeling sleepy or having a break so they perform more tasks within the time limit than human being.

g. Robots can work under water, without air, in the space and in the fire so they are helpful for the human safety.

\section{Disadvantages of robots}

a. Robots need supply of power for doing any task which takes lot of money.

b. Robots lead the human being toward unemployment because lot of factories replaces the human with robots. c. Robots need lot of money for their maintenance and repairing.

d. Robots can store lot of data but their intelligence is not like humans to access so these are not effective as human brain are.

e. Robots have no intelligence or emotions so they have not built any goals in their life in the result they are unable to improve their work efficiency or job.

\section{Other differences}

The robots can move, talk, walk and perform many other tasks seems like human but these robots have not all features like human being. The main difference between both is to produce their generation. Robots cannot' reproduce themselves like human. They have no emotions so have not humanity in it which motivate a human to help others without any interest or greed. Robots have artificial intelligence so they can solve the problems more accurately than human but they cannot solve those problems which are not in their programming disk. They also not make their goals because robots have not determination to do anything new. Robots can achieve the information from very dangerous places but this information can uses by any person even by a wrong person because robots have no decision power to know about right and wrong, their right and wrong only depends on their program that feed in them.

\section{Conclusion}

At the end authors conclude that the robots are a way to make human life easy and effective but there is always the need of human being because there is no alternate of human brain. Human brain is the only which can produce, make, solve, and invent the new things which no any robot have. So there is a need to give employment to human being rather than a robot. Because $\mathrm{He}$ is a human who invent a robot not a robot who create the human. So if we want more inventions, more advancements and more development in the world then we need human. Robot can be used for working on some idea for getting results more quickly and accurately but there is always need a human to give an idea for development in any way of life. We also need human to share, to generate new things and to produce more human generations in the world.

\section{Acknowledgements}

None.

\section{Conflict of interest}

The author declares there is no conflict of interest.

\section{References}

1. Automaton. Free Merriam-Webster Dictionary.

2. Heba Soffar. Advantages and disadvantages of using robots in our life. 2016. 